\title{
Alcohol Exacerbates LPS-Induced Fibrosis in Subclinical Acute Pancreatitis
}

\author{
Haitao Gu, ${ }^{*}$ Franco Fortunato, ${ }^{*}$ Frank Bergmann, ${ }^{\dagger}$ Markus W. Büchler, ${ }^{*}$ David C. Whitcomb, ${ }^{\ddagger}$ and Jens Werner*
}

From the Department of General, Visceral, and Transplantation Surgery* and the Institute of Pathology, ${ }^{\dagger}$ University Clinic Heidelberg, Heidelberg, Germany; and the Department of Gastroenterology, ${ }^{\ddagger}$ University of Pittsburgh, Pittsburgh, Pennsylvania

Accepted for publication

July 12, 2013.

Address correspondence to Franco Fortunato, Ph.D., Department of General, Visceral and Transplantation Surgery, University Clinic Heidelberg, Im Neuenheimer Feld 110, D-69120 Heidelberg, Germany. E-mail: franco.fortunato@med.uniheidelberg.de.

\begin{abstract}
The role of pancreatic acinar cells in initiating fibrogenic responses during the early stages of alcoholic acute pancreatitis has not been evaluated. We investigated the ability of injured acinar cells to generate pancreatic fibrosis in acute pancreatitis. Rats were fed either an ethanol-containing or control diet over 14 weeks and euthanized 3 or 24 hours after a single lipopolysaccharide injection. Profibrotic transforming growth factor- $\beta$ of acinar cells and pancreatic fibrosis were assessed by immunofluorescence, histological characteristics, and electron microscopy. Human pancreatic tissues were also evaluated. Periacinar cell fibrosis and collagen were exacerbated 24 hours after endotoxemia in alcohol-fed rats. Alcohol exposure exacerbated acinar cell-specific production of transforming growth factor $\beta$ in response to lipopolysaccharide in vivo and in acinar cell-like AR42J cells in vitro. Although a morphological examination showed no visible signs of necrosis, early pancreatic fibrosis can be initiated by little or no pancreatic necrosis. Transforming growth factor $\beta$ was also significantly increased in human acinar cells from patients with acute/recurrent pancreatitis compared with chronic pancreatitis tissue. Alcohol exacerbates lipopolysaccharide-induced pancreatic fibrosis during the early onset of mild, subclinical, acute pancreatitis. We suggest that multiple, subclinical, acute pancreatitis episodes can accumulate in fibrosis during the development of chronic pancreatitis, even if there is no history of acute pancreatitis. (Am J Pathol 2013, 183: 1508-1517; http://dx.doi.org/10.1016/j.ajpath.2013.07.023)
\end{abstract}

Experimental models of alcoholic pancreatitis have shown that lipopolysaccharide (LPS) exacerbates the development of alcoholic acute pancreatitis (AP) and that exposure to multiple applications of LPS results in fibrogenesis and chronic pancreatitis $(\mathrm{CP}){ }^{1-4}$ We recently reported that the combination of alcohol and endotoxemia attenuated the apoptosis response, disrupted autophagy signaling, and enhanced pancreatic injury, causing an early onset of AP, which supports clinical observations that episodes of acute and recurrent pancreatitis can progress to $\mathrm{CP} .{ }^{2,4}$ Alcoholics seem more susceptible to AP, a condition associated with an earlier onset, more frequent episodes, and an increased risk of CP. ${ }^{5-8}$ Alcoholic patients also have elevated serum LPS concentrations, which have been associated with the severity of alcoholic AP., 90

Previously accepted consensus definitions of AP and CP postulate that these two clinical syndromes are independent of one another. ${ }^{11}$ Episodes of AP in patients with alcoholic CP are thought to be complications of underlying subclinical
$\mathrm{CP} .^{12} \mathrm{~A}$ popular alternate hypothesis is the necrosis-fibrosis sequence model, in which pancreatic fibrosis and CP occur by recurrent episodes of AP with localized pancreatic necrosis. ${ }^{13,14}$ However, many patients with $\mathrm{CP}$ have no history of clinical AP. Because clinical data and tissue specimens are lacking from patients with AP, in particular from subclinical cases, it remains elusive if subclinical AP episodes can accumulate fibrosis during the development of CP.

According to the necrosis-fibrosis hypothesis, the initial event inducing pancreatic fibrogenesis would require either acinar or ductal cell death by necrosis, followed by tissue destruction and fibrosis. ${ }^{5}$ Thus, pancreatic fibrosis and CP would result from recurrent episodes of AP accompanied by localized pancreatic necrosis. ${ }^{15}$ In this case, acinar cell

Supported by a German Research Foundation (DFG) Excellence Initiative Frontier award (F.F.) and the University Clinic Heidelberg (M.W.B., D.C.W., and J.W.).

H.G. and F.F. contributed equally to this work. 
death through necrosis would be required to initiate a strong inflammatory response, followed by immune cell infiltration and the activation of pancreatic stellate cells (PSCs). ${ }^{16-18}$ Experimental research has shown that activation of PSCs is required to initiate fibrogenesis and $\mathrm{CP} .{ }^{17-20}$ Binding of the profibrogenic transforming growth factor $\beta$ (TGF- $\beta$ ) activates PSCs, resulting in their proliferation, the production and deposition of collagen $1 \mathrm{~A} 1$, and pancreatic fibrogenesis. ${ }^{18,21}$ TGF- $\beta$, a multifunctional cytokine and one of the most investigated profibrogenic factors, is elevated in patients with acute necrotizing pancreatitis and participates in pancreatic fibrogenesis. ${ }^{22-27}$

The role of pancreatic acinar cells in initiating fibrogenic responses during the early stages of AP has not been precisely determined. Because various cell types within the pancreas, including PSCs and the influence of other organs, may modulate TGF- $\beta$ expression during the course of AP, we have attempted to clarify the origin of TGF- $\beta$ during the early onset of AP using rat and human samples. We also have analyzed whether acinar cells are the first responders to alcohol and LPS and are able to generate profibrogenic responses in this model of subclinical pancreatitis.

\section{Materials and Methods}

\section{Animals and Reagents}

Male Sprague-Dawley rats were purchased from Charles River (Sulzfeld, Germany). All experiments were approved by the Institutional Animal Care and Use Committee in accordance with the guidelines of the University of Heidelberg (Heidelberg, Germany) and the Federal Presiding Board of Animal Care (Karlsruhe, Germany).

Antibodies were selected according to proven functionality in formalin-fixed, paraffin-embedded (FFPE) tissue sections by the seller or by publication records. Mouse monoclonal anti- $\alpha$-smooth muscle actin (SMA; sc-32251) and anti- $\alpha$-amylase (sc-46657) were purchased from Santa Cruz, Biotech (Heidelberg). Mouse monoclonal anticollagen 1A1 (ab6308) and rabbit anti-TGF- $\beta$ (ab66043) were purchased from Abcam (Cambridge, UK). All antibodies were suitable for rat and human samples. Secondary anti-rabbit $\mathrm{Cy} 3$ or Cy5-conjugated and anti-mouse Cy5conjugated antibodies were obtained from Medac $\mathrm{GmbH}$ (Wedel, Germany). PCR primers for collagen were purchased from Eurofins MWG Operon (Ebersberg, Germany). All other chemicals were from Sigma-Aldrich (Deisenhofen, Germany), if not stated otherwise.

\section{Experimental Design}

Male rats, weighing 150 to $175 \mathrm{~g}$, were fed a commercially available liquid diet, according to Lieber and DeCarli ${ }^{28}$ (BioServ, Frenchtown, NJ), containing either ethanol (6\% final concentration, equivalent to $36 \%$ of caloric intake in 15 rats) or maltose-dextrin (pair-fed group in 15 rats) in isocaloric amounts, as described previously. ${ }^{2}$ After 14 weeks of feeding the control or alcohol diet, Gram-negative

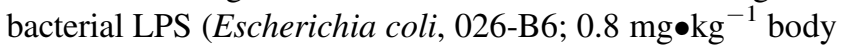
weight, i.v.) or vehicle control (sterile saline) was injected once i.v. Rats were divided into six groups each, with five animals per group: pair-fed (PF) $(n=5)$ and alcohol-fed controls $(n=5)$, and PF or alcohol-fed animals injected with LPS, sedated at 3 or 24 hours after LPS injection $(n=5)$ with pentobarbital (Nembutal, $60 \mathrm{mg} \bullet \mathrm{kg}^{-1}$ body weight, i.p.) (Nembutal; Ovation Pharm, Deerfield, IL). The whole pancreas was resected, immersed in liquid nitrogen, and stored at $-80^{\circ} \mathrm{C}$.

\section{Human Subjects}

The study was approved and renewed in August 2012 by the Ethics Committee of the University Medical Faculty of Heidelberg. Human pancreatic tissues were obtained from patients attending the Department of Surgery of our university. In detail, the series comprised three cases of acute pancreatitis (AP)/recurrent acute pancreatitis (RAP), obtained from three men aged 60, 64, and 70 years (average age, 64 years). Alcohol consumption was known in one of these patients. The remaining two patients did not have a reported history of alcohol use, and pancreatitis occurred after low cardiac output syndrome following heart transplantation and for unknown idiopathic reasons in one case each. The CP group consisted of five male and two female patients, aged between 52 and 70 years (average age, 58 years). A history of alcoholism was known in five of these patients; one case represented obstructive pancreatitis due to impn-related pancreatic cancer, and in one patient the $\mathrm{CP}$ was idiopathic. The indications for pancreatic head resections were pain- and CP-related complications in organs neighboring the pancreas, such as common bile duct stenosis, main pancreatic duct stenosis, vascular obstruction, and/or duodenal obstruction. Normal human pancreatic tissues were obtained from one female and two male patients (aged 31, 59, and 72 years; average age, 54 years) who were free of pancreatic disease (through an organ donor program in which there were no candidates for pancreatic transplantation).

With one exception, all samples (AP, CP, and controls) were taken from the pancreatic head. One case was taken from the pancreatic tail. Freshly removed tissue samples were immediately fixed in $4 \%$ buffered formalin.

To determine the stage of the disease, the fibrosis and severity and activity of the inflammation were microscopically evaluated according to previously described criteria. ${ }^{29}$ The control samples revealed regular pancreatic tissue, consisting of lobules of acinar cells, ducts, and scattered islets. Focally, mild fibrosis was detected in two cases (scores 0,1 , and 2). There was no significant inflammation (severity score, 0 ; activity score, 0 ). In chronic pancreatitis, periductal, interlobular, and intralobular fibrosis was seen to various extents (scores, 4 to 12; average score, 6.9). 
Furthermore, the pancreatic tissues displayed mild to moderate chronic inflammation with scattered lymphocytes (severity scores, 1 to 2 ; average score, 1.4). The activity of the inflammation was mild in three cases (score, 1), mild to moderate in one case (score, 2), and absent (score, 0) in three cases (average score, 0.7). Necrosis was generally lacking. In acute/recurrent pancreatitis, the samples showed multiple areas of fatty necrosis and partially dense infiltrates of neutrophils (activity scores, 2, 3, and 3; average score, 2.7) and lymphocytes (severity scores, 2, 2, and 3; average score, 2.3). Mild to moderate, diffuse interlobular and intralobular fibrosis was seen in one of these cases (score, 9), whereas the remaining two cases presented only scarce, focal fibrosis (scores, 2 and 3).

\section{Immunofluorescence}

Immunofluorescence was conducted using pancreatic FFPE tissue sections ( $5 \mu \mathrm{m}$ thin) obtained from rat and human specimens and processed as described. ${ }^{2}$ Primary antibodies were incubated in antibody diluent (Dako, Hamburg, Germany) for 30 minutes at $37^{\circ} \mathrm{C}$ in a dark humidified chamber, followed by several steps of washing. Sections were then incubated with anti-mouse-Cy5 and/or anti-rabbit-Cy3 secondary antibodies for 30 minutes at $37^{\circ} \mathrm{C}$ in the dark, also followed by several steps of washing and incubation with DAPI for 20 minutes. The sections were mounted in Fluoromount-G Reagent (SouthernBiotech, Birmingham, AL). Ten images per slide/sample, with typically 1700 to 3500 cells $(63 \times$ objective) were captured using a Zeiss Axiovert 200M microscope (Zeiss, Göttingen, Germany) equipped with DAPI, Cy3, and Cy5 filters (Chroma Technology, Rockingham, VT), as described recently. ${ }^{2}$ Briefly, standardized automatic acquisition was performed by AQuest software version 2.2 (TissueGnostics, Vienna, Austria) controlling filters, exposure time, camera (PCO, Kehlheim, Germany), and motor stage (Märzhäuser, Wetzlar, Germany). All images were processed using (TissueGnostics), allowing the quantitation of the total cell numbers from DAPIpositive cells and quantitation of target-positive cells. In fluorescence-activated cell sorter (FACS)-like scattergrams, the cells were plotted according to their Cy3 and Cy5 immunofluorescence (IF) intensity versus their DAPI intensity from 10 randomly captured images. The IF-positive cells were gated in the scattergrams, according to negative controls (no primary antibody), and the fluorescence intensity was expressed as percentage of the intensity of the DAPI staining, as described recently. ${ }^{2}$ Likewise, cellular colocalization of rat and human $\alpha$-amylase with TGF- $\beta$ was analyzed, and cells were counted in the same way, with the software-supported colocalization analysis.

\section{IHC Data}

Immunohistochemistry (IHC) was also conducted using pancreatic FFPE tissue sections ( $7 \mu \mathrm{m}$ thin) obtained from rat and human specimens, which were processed using mouse monoclonal anti- $\alpha$-SMA, as described previously., ${ }^{2,3}$

\section{In Vitro Alcohol and LPS Stimulation}

The rat pancreatic acinar-like AR42J cell line was purchased from ATCC (Manassas, VA), and cells were grown in Ham's F-12 medium supplemented with $20 \%$ heat-inactivated fetal calf serum, $50 \mathrm{U} / \mathrm{mL}$ penicillin $\mathrm{G}, 50 \mu \mathrm{g} / \mathrm{mL}$ streptomycin, and $2.5 \mathrm{mg} / \mathrm{mL}$ plasmocin. The cells were maintained at $37^{\circ} \mathrm{C}$ in a humidified atmosphere of $95 \%$ air and $5 \% \mathrm{CO}_{2}$. AR42J cells were suspended and plated onto sterile coverslips (Nalgene; ThermoScientific, Waltham, MA) in 6-well tissue culture plates at a density of 300,000 cells per well and incubated for 24 hours. The cells were treated with various doses of ethanol (50 to $400 \mathrm{mmol} / \mathrm{L}$ ), with or without $10 \mu \mathrm{g} / \mathrm{mL}$ LPS, for another 24 hours. After fixation with $2 \%$ paraformaldehyde for 30 minutes in the dark at room temperature, the cells were IF stained for TGF- $\beta$ and $\alpha$-amylase, as described. All cells were mounted in Fluoromount-G Reagent, and a minimum of 10 images per treatment were captured and analyzed using the same microscope and software, as described. ${ }^{2}$ All in vitro experiments were repeated at least three times.

\section{Pancreatic Histological Characteristics}

Pancreata were cut into serial sections ( $4 \mu \mathrm{m}$ thick) from $4 \%$ FFPE blocks. Sections were stained with H\&E and examined by a pathologist (F.B.) who was not aware of the sample identity. Tissue sections $(n=4)$ were examined for parenchymal edema, acinar cell vacuolization, necrosis, inflammatory cell infiltration, and hemorrhage, and analyzed in 10 randomly selected fields with the aid of the Olympus BX40 microscope camera system (Hamburg, Germany), as described previously. ${ }^{2}$

\section{Electron Microscopy Observation}

Frozen pancreatic tissue $(n=4)$ was fixed in ice-cold $1.5 \%$ paraformaldehyde and embedded in Epon 812 (SigmaAldrich). Ultrathin sections were prepared for microscopy according to a routine procedure described previously. ${ }^{2}$ Briefly, areas within scanned images of electron micrographs were measured using a Metamorph-tracing semiautomatic counting tool. The fibrosis index was determined by an automatic computer-assisted tracing tool in relation to the entire traced area using analySIS 3.2 Software (Soft Imaging System, Munster, Germany) and expressed in $\mathrm{nm}^{2}$ fibrosis in relation to the entire region. All areas were calibrated to scale bars on the micrographs and expressed as a percentage of the entire region measured, and were digitally evaluated in four animals per group $(n=4)$ in 100 randomly selected $45 \times 45-\mu \mathrm{m}$ fields per animal, as described. ${ }^{2}$ Electron microscopic (EM) analysis was performed using an analytical device (EM 902; Zeiss, Oberkochen, Germany) 
A

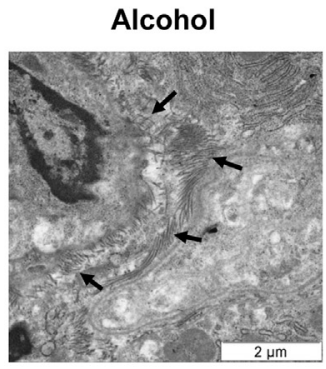

Alcohol plus LPS

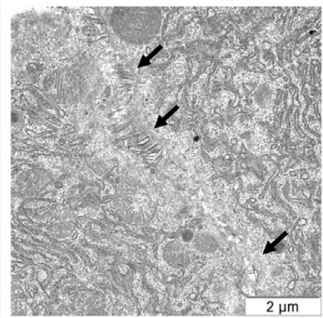

C

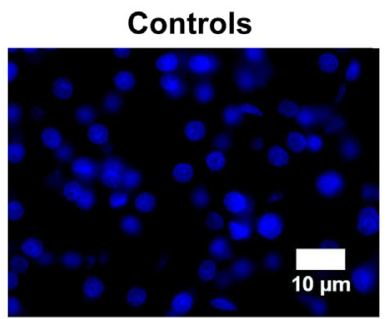

DAPI/Collagen
Alcohol + 24 h LPS

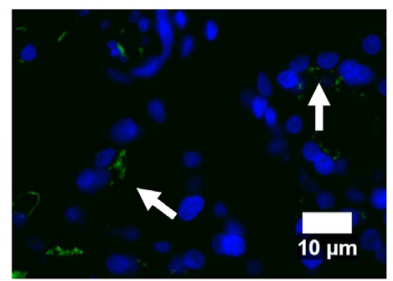

B

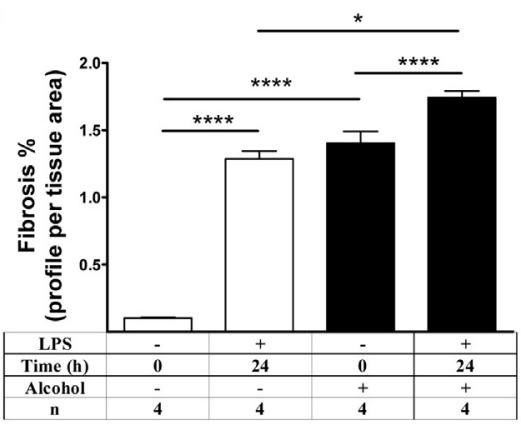

D

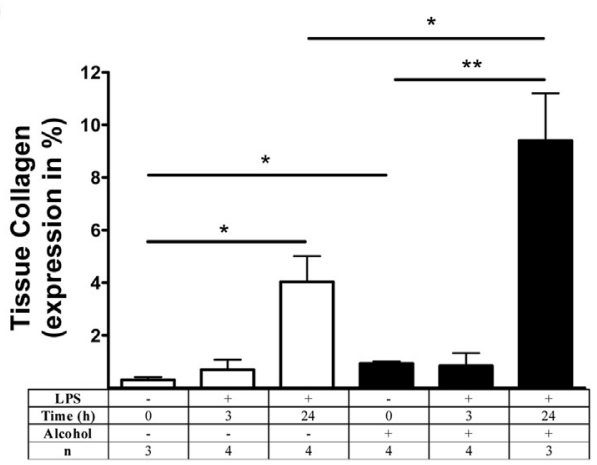

F

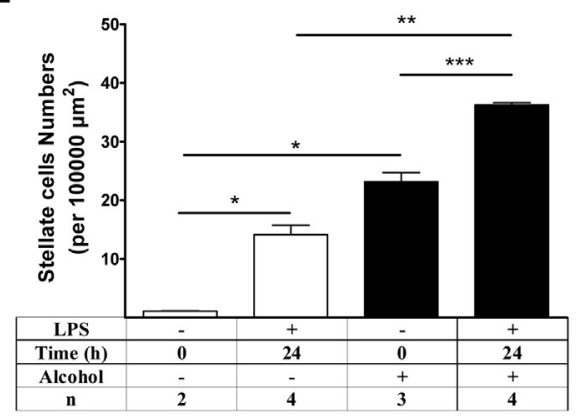

Figure 1 LPS exacerbates pancreatic fibrosis during the early onset of subclinical acute alcoholic pancreatitis. A: Representative EM images of pancreatic acinar cells exposed to alcohol for 14 weeks alone or 24 hours after LPS treatment. Arrows indicate typical periacinar cell collagen fibers. B: The quantitation of collagen was plotted as means \pm SEM of three to four animals per group. C: Representative IF images of PF control and alcohol-fed (EtOH) rat tissue 3 and 24 hours after LPS stained for collagen 1A (Cy3, green) and DAPI (blue) using an IF microscope. Original magnification, $\times 63$. Arrows indicate periacinar cell collagen (green) deposition. D: Collagen expression was plotted as means \pm SEM of three to four animals per group. E: Representative IHC images of PF and $\mathrm{EtOH}$ rats 24 hours after LPS stained for $\alpha$-SMA (brown). Arrows indicate potential $\alpha$-SMA-positive stellate cells. F: Quantitation of EM numbers of pancreatic stellate cells in pancreatic tissue. Graph represents the tissue analysis of the numbers of stellate cells obtained from PF and EtOH rats 24 hours after LPS. Quantitation values were plotted as means \pm SEM for three animals per group. ${ }^{*} P \leq 0.05,{ }^{* *} P \leq 0.01,{ }^{* * *} P \leq 0.001$, and ${ }^{* * * * P} \leq 0.0001$.

coupled with a Pro-Scan digital camera (Troendle, Munich, Germany).

\section{Real-Time PCR}

Frozen whole pancreatic tissue was powdered in the presence of liquid nitrogen and extracted using TRIzol-Reagent (Life Technologies-Invitrogen, Carlsbad, CA). Total RNA (5 $\mu \mathrm{g})$ was reverse transcribed using SuperScript II (Life Technologies, Invitrogen), according to the manufacturer's instructions. A real-time PCR was analyzed on a TaqMan 7000
(Applied Biosystems, Foster City, CA) under standard conditions, using the TaqMan system. The calculated cycles were subtracted from the 18s RNA (Applied Biosystems) reference, which was always included on the same plate. The difference in cycles was then calculated and expressed as previously relative transcript levels $\left(2^{\Delta \Delta}\right)$. The TaqMan primer and probe sequences for collagen-3a-1 were designed using ABI primer express software: forward: 5'-GTGAAAGAGGATCTGAGGGCTC-3', reverse: 5'-GAGTTCAGGGTGGCAGAATTT-3', and probe: $5^{\prime}$-TGCTGCCATTGCTGGAGTTGGA- $3^{\prime}$. Real-time PCR was analyzed as described. ${ }^{30}$ 
A

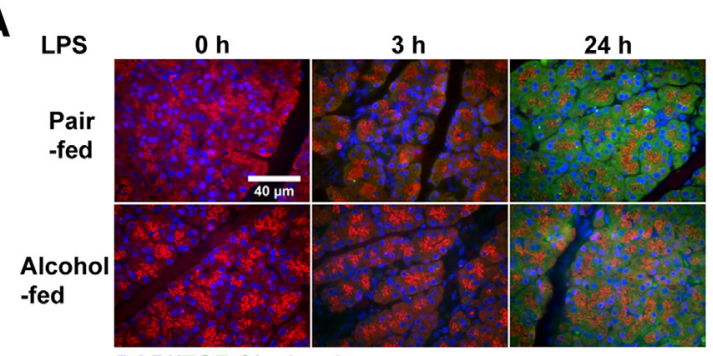

DAPI/TGF- $\beta / \alpha-A m y l a s e$
B

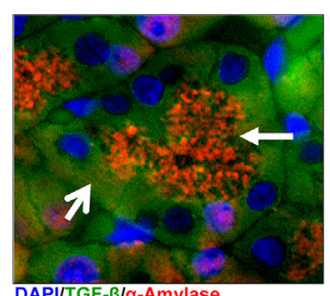

DAPIITGF-Bla-Amylase
C

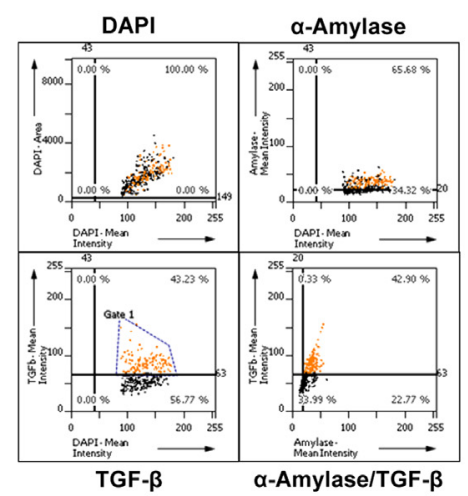

D

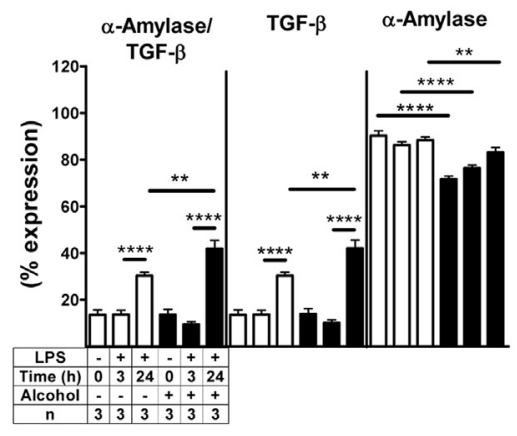

E

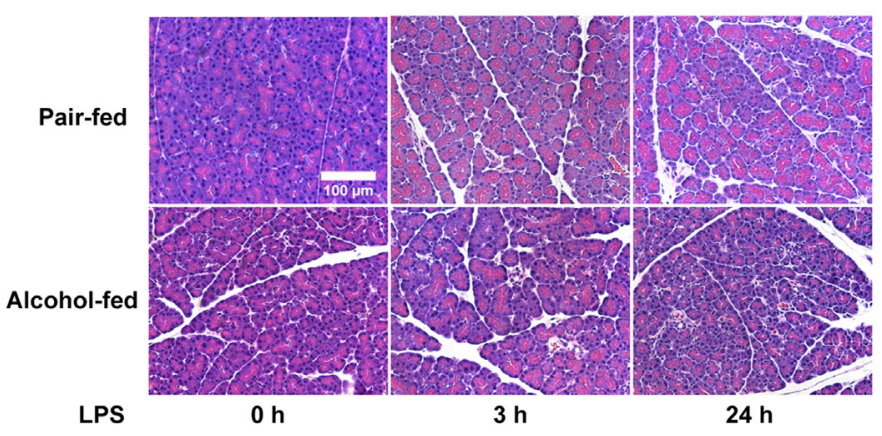

$\mathbf{F}$

No LPS

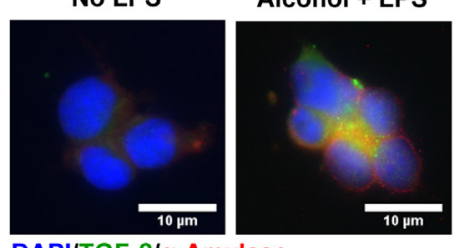

DAPI/TGF- $\beta / \alpha-A m y l a s e$
Alcohol + LPS
G

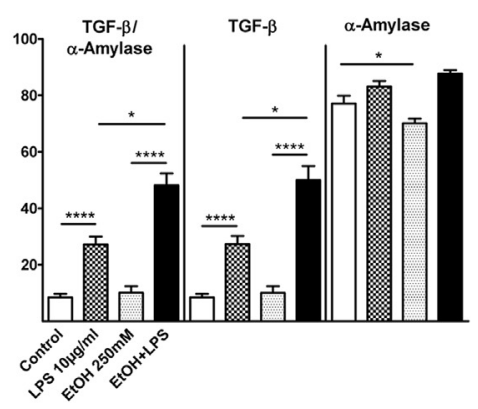

Figure 2 Alcohol enhanced early LPS-induced TGF- $\beta$ production in pancreatic acinar cells. A: Representative IF images showing colocalization of TGF- $\beta$ (Cy3, green), $\alpha$-amylase (Cy5, red), and DAPI (blue) in rat pancreatic tissue from PF controls and alcohol-fed (EtOH) animals 3 and 24 hours after LPS treatment, respectively. B: Higher magnification of an EtOH rat tissue section staining 24 hours after LPS treatment shows that $\alpha$-amylase (Cy5, red) is mainly expressed in the apical region of the acinar cells (arrow), whereas TGF- $\beta$ (Cy3, green) is mainly expressed in the basolateral region of the acinar cells (open arrow). C: Representative tissue-FACS scattergram analysis obtained from TGF- $\beta$ and $\alpha$-amylase colocalization quantitation in EtOH animals 24 hours after LPS for DAPI-positive nuclei/cells, TGF- $\beta$ /DAPI quantitation, $\alpha$-amylase/DAPI quantitation, and TGF- $\beta$ and $\alpha$-amylase colocalization quantitation, shown as yellow dots representing TGF- $\beta$-positive cells in gate 1. D: TGF- $\beta$ and $\alpha$-amylase colocalization quantitation plotted as means \pm SEM for three animals per group. E: Representative H\&E-stained pancreatic tissue images of PF and EtOH rat tissue section 3 and 24 hours after LPS treatment, respectively. Some edema and vacuolization are visible in EtOH animals 24 hours after LPS. No visible necrosis is recognizable. F: In vitro IF quantitation for TGF- $\beta$ (Cy3, green) and $\alpha$-amylase (Cy5, red) and DAPI (blue) in AR42J cells exposed to alcohol and LPS. G: Colocalization quantitation was plotted as means \pm SEM for at least three independent experiments. ${ }^{*} P \leq 0.05,{ }^{* *} P \leq 0.01$, and ${ }^{* * * * P} \leq 0.0001$. 
Table 1 Cells in 10 Randomly Captured Rat Pancreatic Tissue Images Stained for TGF- $\beta$

\begin{tabular}{llllrlr}
\hline TGF- $\beta$ & PF & PF +3 hours LPS & PF +24 hours LPS & EtOH & EtOH +3 hours LPS & EtOH +24 hours LPS \\
\hline Total cells & 5215 & 6470 & 5867 & 5697 & 7977 & 7959 \\
$\alpha$-Amylase cells & $5166(99)$ & $5935(91)$ & $5483(93)$ & $3987(70)$ & $6347(79)$ & $7014(88)$ \\
TGF- $\beta$ cells & $780(14.8)$ & $985(14.7)$ & $2082(35)$ & $923(16)$ & $756(9)$ & $3485(41)$ \\
$\alpha$-Amylase/TGF- $\beta$ cells & $780(14.8)$ & $985(14.7)$ & $2079(35)$ & $900(15)$ & $695(8.3)$ & $3469(41)$ \\
Other TGF- $\beta$ cells & $0(0)$ & $0(0)$ & $3(0)$ & $23(0.3)$ & $61(0.7)$ & $16(0.2)$ \\
\hline
\end{tabular}

Values are given as number (percentage) unless otherwise indicated. Cells were counted with the aid of the Tissue Quest Microscope and Tissue-FACS analysis software version 3.0.1 (TissueGnostics, Vienna, Austria). All $\alpha$-amylase-negative cells positive for TGF- $\beta$ were extremely low.

EtOH, alcohol; LPS, lipopolysaccharide; PF, pair-fed; TGF- $\beta$, transforming growth factor $\beta$.

\section{Statistical Analysis}

Statistical analysis was performed by using the analysis of variance, followed by the Student's $t$-test for each experimental group treated with LPS or vehicle. Results were considered significant when $P<0.05$, and were reported as means \pm SEM or as normalization to control samples. Statistical calculations were performed using software from GraphPad Prism 5 (La Jolla, CA).

\section{Results}

Pancreatic Fibrosis Is Established at the Early Onset of Subclinical Pancreatitis

We investigated whether LPS induced subclinical pancreatic fibrogenesis in the alcohol-fed animals. We investigated fibrosis by applying electron microscopy and determining the collagen deposition (Figure 1A). EM collagen deposition increased significantly in pair-fed controls (12-fold) and alcohol-fed animals (15-fold), 24 hours after LPS. Alcohol alone increased collagen deposition by 14 -fold when compared with pair-fed animals, whereas the combination of alcohol and LPS increased collagen by 1.355 -fold compared with LPS alone (Figure 1B). We did not find collagen deposition 3 hours after LPS. We confirmed this surprising result by tissue IF staining for collagen staining (Figure 1C). Collagen level significantly increased by 13fold in pair-fed controls and by 25 -fold in alcohol-fed animals 24 hours after LPS compared with pair-fed controls. Alcohol alone for 14 weeks also increased collagen by threefold, whereas the combination of alcohol and 24-hour LPS increased collagen by 2.3 -fold compared with pairfed controls 24 hours after LPS (Figure 1D). Collagen mRNA expression was confirmed by real-time PCR (Supplemental Figure S1).

IHC is typically used to stain $\alpha$-SMA, a marker for stellate cells. We also observed elevated $\alpha$-SMA expression in alcoholfed animals 24 hours after LPS, indicating more PSCs after alcohol exposure 24 hours after LPS (Figure 1E). In addition, we counted the number of PSCs in the EM samples in a similar manner to determine collagen deposition. In the EM samples, the number of PSCs increased in pair-fed controls by 12 -fold and in the alcohol-fed animals by 32 -fold 24 hours after LPS, whereas alcohol alone increased PSCs by 20 -fold when compared with the PF controls. There was also a 2.5 -fold increase in PSC numbers in the alcohol-fed group compared with pair-fed animals 24 hours after LPS (Figure 1F).

Overall, we were able to show exacerbated pancreatic fibrosis as early as 24 hours after LPS in this model of subclinical acute alcoholic pancreatitis.

\section{Alcohol-Enhanced LPS-Induced TGF- $\beta$ Production in Pancreatic Acinar Cells}

We next determined whether TGF- $\beta$ signaling is altered in the experimental settings because TGF- $\beta$ is the major regulator for fibrogenesis. ${ }^{31}$ We investigated the expression of TGF- $\beta$ and $\alpha$-amylase using double IF staining (Figure 2A). TGF- $\beta$ was found in the basolateral region of the acinar cell, whereas $\alpha$-amylase was found mostly in the apical region, indicating a true distribution within acinar cells (representative shown for TGF- $\beta / \alpha$-amylase) (Figure $2 B$ ). All results were obtained from FACS-like scattergram analysis (Figure 2C). TGF- $\beta$ and $\alpha$-amylase colocalization increased significantly in pair-fed and alcohol-fed animals 24 hours after LPS and was significantly higher in alcohol-fed animals. Almost identical results were obtained for the expression of TGF- $\beta$ alone. Moreover, $\alpha$-amylase expression alone showed a significant reduction after alcohol exposure, suggesting alcohol-reduced $\alpha$-amylase expression (Figure 2D). We confirm our data by counting all evaluated cells in 10 images for TGF- $\beta$ positivity alone or in colocalization with $\alpha$-amylase. Only a few $\alpha$-amylase-negative cells were positive for TGF- $\beta$, indicating that most TGF- $\beta$ s were produced by pancreatic acinar cells in subclinical acute pancreatitis (Table 1).

Pancreatic necrosis is believed to initiate inflammatory responses, fibrogenesis, and pancreatitis, according to the necrosis-fibrosis hypothesis. ${ }^{13}$ Histopathological alterations showed mild edema and little vacuolization 24 hours after LPS in both groups. We did not identify any visible signs of intense acinar cell necrosis in the tissues from pair-fed or alcohol-fed animals either 3 or 24 hours after LPS administration (Figure 2E). In addition, we confirmed that TGF- $\beta$ expression was also evident in acinar-like AR42J cells in vitro (Figure $2 \mathrm{~F}$ ), and increased TGF- $\beta$ and $\alpha$-amylase colocalization has been significantly found after LPS and 
A
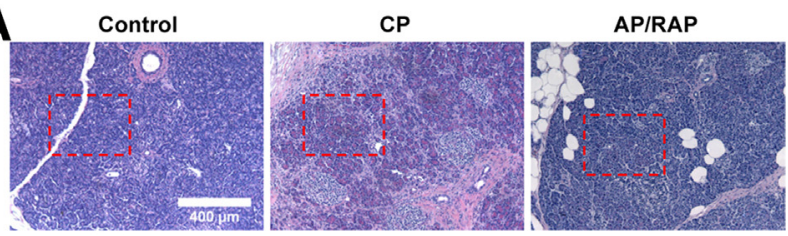

B

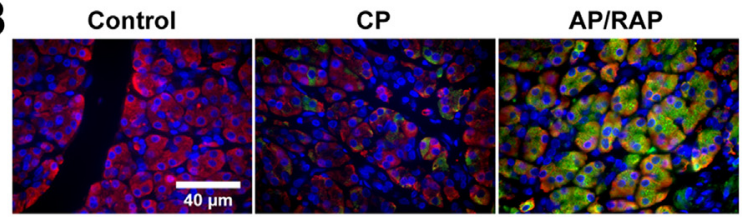

DAPI/TGF- $\beta / \alpha-A m y l a s e$

C

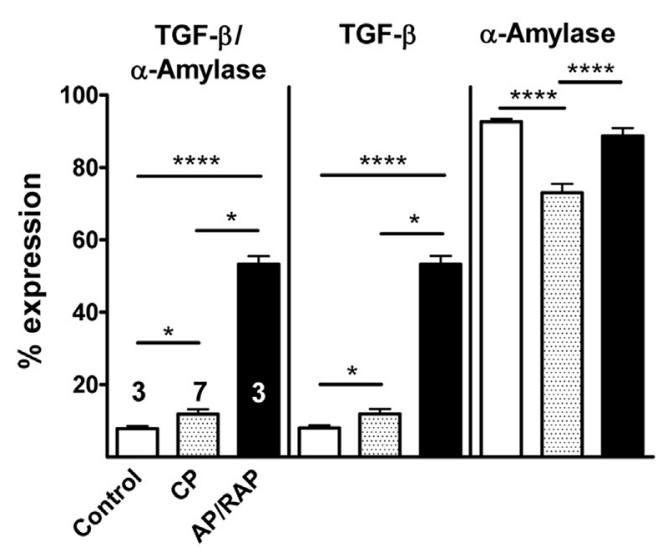

D
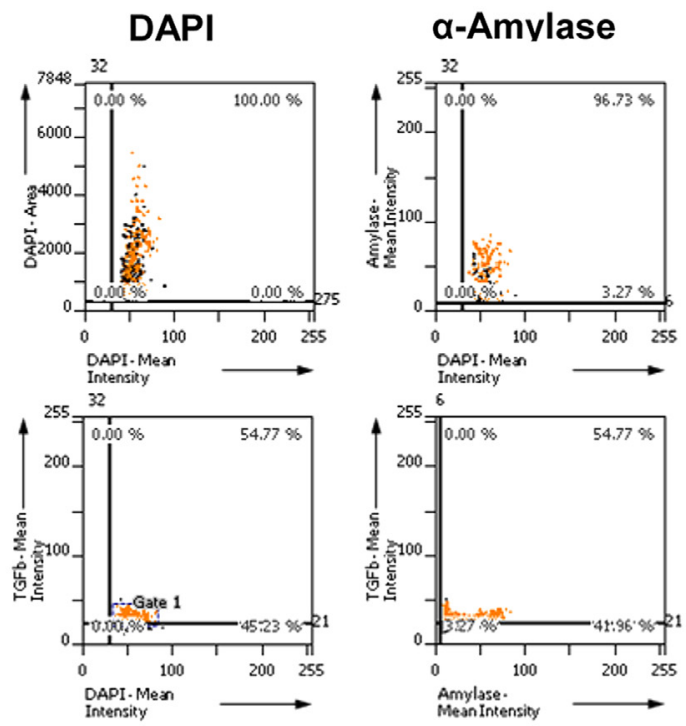

TGF- $\beta$

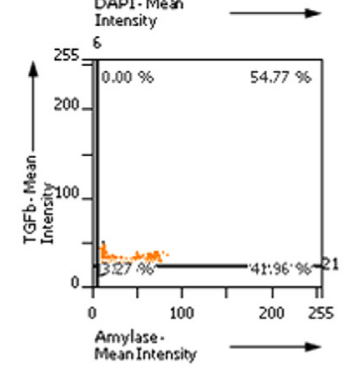

$\alpha$-Amylase/TGF- $\beta$

E
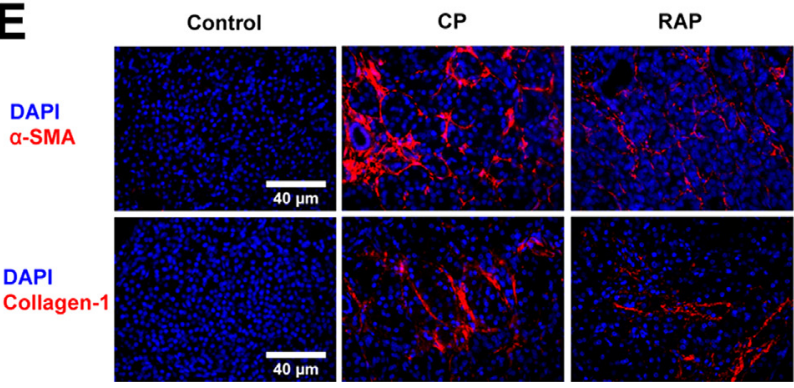

alcohol concentrations ranging from 200 to $400 \mathrm{mmol} / \mathrm{L}$ (Supplemental Figure S2). LPS significantly increased TGF- $\beta$ and $\alpha$-amylase colocalization by 3.2 -fold, which was enhanced with $250 \mathrm{mmol} / \mathrm{L}$ alcohol by 1.8 -fold, indicating that alcohol exacerbated LPS-induced TGF- $\beta$ production in vitro, confirming, in part, the in vivo results (Figure $2 \mathrm{G}$ ). These results strengthened the evidence that TGF- $\beta$ is produced in pancreatic acinar cells during the early onset of AP. We conclude that pancreatic acinar cells were capable of producing profibrogenic TGF- $\beta$, which, in turn, can activate PSCs, collagen production, and fibrogenesis in the early onset of AP.

\section{Human Acinar Cells Expressing Profibrotic TGF- $\beta$ in Acute/Recurrent Pancreatitis Specimens}

We investigated control tissue from donor patients, patients with $\mathrm{CP}$, and AP/RAP specimens to determine the expression of profibrotic TGF- $\beta$ in areas of intact acini structure, with representative images shown in the H\&E-stained tissue sections (Figure 3A). We next determined TGF- $\beta$ and $\alpha$ amylase colocalization by IF in tissue obtained from donor, $\mathrm{CP}$, and RAP patients (Figure 3B). TGF- $\beta$ and $\alpha$-amylase colocalization increased substantially in AP/RAP tissue compared with donor controls by sevenfold and 4.5-fold, respectively, compared with $\mathrm{CP}$ tissue. Similar results were found for TGF- $\beta$ alone, suggesting that acinar cells produce most TGF- $\beta$. $\alpha$-Amylase expression decreased by 1.3 -fold in CP tissue, although $\alpha$-amylase did not significantly change in AP/RAP samples compared with controls (Figure 3C). We confirm the data by counting all evaluated cells in 10 images for TGF- $\beta$ positivity alone or in colocalization with $\alpha$-amylase. Only a few $\alpha$-amylase-negative cells were positive for TGF- $\beta$, indicating that most TGF- $\beta$ was produced by pancreatic acinar cells in acute/recurrent pancreatitis (Table 2). All results were obtained from the representative shown FACS-like scattergram analysis graph (Figure 3D).

We determined the degree of fibrosis by IF staining for collagen- 1 and $\alpha$-SMA in the human samples. $\alpha$-SMA and

Figure 3 TGF- $\beta$ and fibrosis in acinar cells increase in human acute/ recurrent pancreatitis specimens. A: Representative H\&E-stained tissue section images indicating areas of intact acini structures of healthy controls, chronic pancreatitis, and acute/recurrent pancreatitis samples chosen for the IF quantitation. B: Representative IF images and colocalization of TGF- $\beta$ (Сy3, green), $\alpha$-amylase (Cy5, red), and DAPI (blue) in human pancreatic tissue specimens. C: Quantitation of TGF- $\beta$ (Cy3, green) and $\alpha$-amylase (Cy5, red) colocalization plotted as means \pm SEM for human specimens $(n=3$ to 7$)$. ${ }^{*} P \leq$ $0.05, * * * * P \leq 0.0001$. D: Representative tissue-FACS scattergram analysis obtained from TGF- $\beta$ and $\alpha$-amylase colocalization quantitation in human acute/recurrent pancreatitis samples, stained for DAPI-positive nuclei/cells, $\alpha$ amylase/DAPI quantitation, TGF- $\beta$ /DAPI quantitation, and TGF- $\beta$ and $\alpha$ amylase colocalization quantitation, shown as yellow cells in gate 1 . E: The fibrosis markers, $\alpha$-SMA (Cy5, red) and collagen-1A (Cy5, red), were highly expressed in both chronic pancreatitis and human acute/recurrent pancreatitis specimens. 
Table 2 Cells in 10 Randomly Captured Human Pancreatic Tissue Images Stained for TGF- $\beta$

\begin{tabular}{lcll}
\hline TGF- $\beta$ (10 images) & $\begin{array}{l}\text { Control } \\
(n=3)\end{array}$ & $\begin{array}{l}\text { CP } \\
(n=7)\end{array}$ & $\begin{array}{l}\text { AP/RAP } \\
(n=3)\end{array}$ \\
\hline Total cells & 9732 & 19736 & 8223 \\
$\alpha$-Amylase cells & $9359(96)$ & $17,193(87)$ & $8159(99)$ \\
TGF- $\beta$ cells & $911(9.1)$ & $2367(11.8)$ & $4422(53)$ \\
$\alpha$-Amylase/TGF- $\beta$ cells & $905(9)$ & $2351(11.7)$ & $4421(53)$ \\
Other TGF- $\beta$ cells & $6(0)$ & $16(0)$ & $1(0)$ \\
\hline
\end{tabular}

Values are given as number (percentage) unless otherwise indicated. Cells were counted with the aid of the Tissue Quest Microscope and TissueFACS analysis software version 3.0.1 (TissueGnostics, Vienna, Austria). All $\alpha$-amylase-negative cells positive for TGF- $\beta$ were extremely low.

$A P$, acute pancreatitis; $C P$, chronic pancreatitis; RAP, recurrent alcoholic pancreatitis; TGF- $\beta$, transforming growth factor $\beta$.

collagen-1 expressions are known to participate in fibrogenesis and are mainly expressed by activated stellate cells. ${ }^{16,18} \mathrm{We}$ chose tissue areas with almost intact acini structures to determine periacinar fibrosis in the human specimens. Periacinar collagen- 1 and $\alpha$-SMA expressions appeared to be similar in AP/RAP and CP tissues, indicating that during the course of AP/RAP, fibrosis has been established, along with the high expression level of TGF- $\beta$, promoting fibrogenesis in AP/RAP patients (Figure 3E).

We were able to confirm the experimental observation in the clinical specimens and suggest that $\mathrm{CP}$ can be established with little acinar cell necrosis, and pancreatic acinar cells are the first responders in driving fibrogenesis by expressing TGF- $\beta$.

\section{Discussion}

Our findings indicate that rat and human pancreatic acinar cells can produce the most important profibrotic mediator, TGF- $\beta$, in the early onset of acute pancreatitis. We also found that the expression of TGF- $\beta$ was highly enhanced in response to LPS in alcohol-fed rats, indicating that chronic alcohol exposure exacerbated the degree of fibrosis in this experimental model of subclinical AP. The expression profile of TGF- $\beta$ clearly indicates that it is produced during the early response to LPS and found exclusively expressed by acinar cells, because only a few $\alpha$-amylase-negative cells were positive for TGF- $\beta$. In addition, acinar cell-like AR42J cells positive for $\alpha$ amylase-producing TGF- $\beta$ in response to LPS and TGF- $\beta$ expression were also enhanced by alcohol in vitro, confirming the experimental and human results in vivo. Samples from patients with acute/recurrent pancreatitis showed that acinar cells were responsible for the production of TGF- $\beta$, indicating that human pancreatic acinar cells are also the major source of profibrotic TGF- $\beta$ in patients with AP. Thus, acinar cells are the major source of TGF- $\beta$ after early pancreatic injury and during the early course of subclinical AP, with these responses involving the toll-like receptor-4 signaling pathway in acinar cells.

Human and rat pancreatic acinar cells express toll-like receptor-4, indicating that acinar cells are capable of responding directly to LPS. ${ }^{1,32-34}$ Our results are the first to show that pancreatic acinar cells express TGF- $\beta$, and participate in the initiation of acute pancreatitis-associated early fibrogenesis. Although Vonlaufen et $\mathrm{al}^{1}$ have shown that multiple LPS injections cause enhanced fibrosis in alcoholic-fed rats, this study did not evaluate fibrosis or TGF- $\beta$ production at the early onset of AP in rats or human specimens. Data suggest that the fibrotic process can be initiated by a subclinical AP event and that acinar cells are the first responders to pancreatic injury, producing profibrogenic TGF- $\beta$, results observed in both human and rat samples. These results also indicated that the profibrotic TGF- $\beta$ production by acinar cells seems to be a common mechanism in acute pancreatitis and is not associated primarily by acute alcoholic pancreatitis.

Subsequently, TGF- $\beta$ activates PSCs and promotes collagen deposition and fibrosis, as described by the necrosisfibrosis hypothesis. ${ }^{5,13}$ TGF- $\beta$ colocalized with $\alpha$-amylase in pancreatic acinar cells, indicating that these cells produce TGF- $\beta$ during the early stages of AP. Our results are in agreement with recent findings in transgenic models of $\mathrm{CP}$, showing that elastase-promoter-targeted acinar cells with defective TGF- $\beta$ signaling prevent pancreatic fibrogenesis, and that the overexpression of Smad6 exacerbates pancreatic fibrogenesis. ${ }^{25,26,35}$ Thus, acinar cells are the major source of TGF- $\beta$ during the early onset of AP, activating PSCs and, subsequently, pancreatic fibrosis. Although PSCs were also apparently responsive to alcohol and LPS, the findings indicate that PSCs do not play a significant role in the initiation of fibrogenesis during the early onset of AP.

Fibrosis varies in degree and may resolve rapidly during recovery from acute subclinical injury in the experimental model. The persistence of mild injury signals or acinar cell stress may be responsible for more severe fibrosis, as observed in patients with alcoholic CP and in experimental models. ${ }^{36}$ Animal models are critical for evaluating early tissue responses to pancreatic injury, because pancreatic tissue is, in general, not available from humans at these early time points. However, we did confirm periacinar cell fibrosis and increased TGF- $\beta / \alpha$-amylase colocalization in acinar cells only from AP/RAP patients, strengthening our experimental results. The detection of TGF- $\beta$ expression in intact acini in patients with AP/RAP or CP and in the rat model, in the absence of visible areas of necrotic tissue, indicates that $\mathrm{CP}$ can be established by subclinical alcoholic AP. Therefore, clinicians may consider that fibrosis in chronic pancreatitis can develop without a history of clinical acute pancreatitis and that the development of fibrosis can be established by multiple subclinical acute pancreatitis cases.

Acinar cells respond to injury-associated stimuli, such as endotoxin, by producing TGF- $\beta$. This, in turn, activates PSCs, fibrogenesis, and the infiltration of immune cells, in accordance with clinical findings that $\mathrm{CP}$ is often 
established without either clinically acute or recurrent episodes of pancreatitis.

\section{Acknowledgments}

We thank Drs. David J. Hackam, Ion Deaciuc, and Nathalia Giese for their help; Karin Ruf, Brunhilde Bentzinger, and Peter Rieger for technical assistance; and the Pathophysiology Department at the University of Heidelberg for the immunofluorescence microscopy support.

Study concept, analysis, and statistics: H.G. and F.F.; pathological analysis: F.B; supervision and funding: F.F., M.W.B., D.C.W., and J.W.; and manuscript writing: F.F., M.W.B., D.C.W., and J.W.

\section{Supplemental Data}

Supplemental material for this article can be found at http://dx.doi.org/10.1016/j.ajpath.2013.07.023.

\section{References}

1. Vonlaufen A, Xu Z, Daniel B, Kumar RK, Pirola R, Wilson J, Apte MV: Bacterial endotoxin: a trigger factor for alcoholic pancreatitis? evidence from a novel, physiologically relevant animal model. Gastroenterology 2007, 133:1293-1303

2. Fortunato F, Bürgers H, Bergmann F, Rieger P, Büchler MW, Kroemer G, Werner J: Impaired autolysosome formation correlates with Lamp-2 depletion: role of apoptosis, autophagy, and necrosis in pancreatitis. Gastroenterology 2009, 137:350-360, 360.e1-5

3. Fortunato F, Deng X, Gates LK, McClain CJ, Bimmler D, Graf R, Whitcomb DC: Pancreatic response to endotoxin after chronic alcohol exposure: switch from apoptosis to necrosis? Am J Physiol Gastrointest Liver Physiol 2006, 290:G232-G241

4. Fortunato F, Kroemer G: Impaired autophagosome-lysosome fusion in the pathogenesis of pancreatitis. Autophagy 2009, 5: 850-853

5. Kloppel G: Progression from acute to chronic pancreatitis: a pathologist's view. Surg Clin North Am 1999, 79:801-814

6. Lankisch PG, Brinkmann G, Maisonneuve P, Lowenfels AB: The natural history of acute pancreatitis: a long-term population-based study. Pancreas 2007, 35:410

7. Nojgaard C, Becker U, Matzen P, Andersen JR, Holst C, Bendtsen F: Progression from acute to chronic pancreatitis: prognostic factors, mortality, and natural course. Pancreas 2011, 40:1195-1200

8. Reszetow J, Hac S, Dobrowolski S, Stefaniak T, Wajda Z, Gruca Z, Sledzinski Z, Studniarek M: Biliary versus alcohol-related infected pancreatic necrosis: similarities and differences in the follow-up. Pancreas 2007, 35:267-272

9. Bode C, Kugler V, Bode JC: Endotoxemia in patients with alcoholic and non-alcoholic cirrhosis and in subjects with no evidence of chronic liver disease following acute alcohol excess. J Hepatol 1987, 4:8-14

10. Bode JC, Bode C: Alcohol, the gastrointestinal tract and pancreas, [in German]. Ther Umsch 2000, 57:212-219

11. Sarles H, Sarles JC, Camatte R, Muratore R, Gaini M, Guien C, Pastor J, Le Roy F: Observations on 205 confirmed cases of acute pancreatitis, recurring pancreatitis, and chronic pancreatitis. Gut 1965 , 6:545-559
12. Ammann RW: A clinically based classification system for alcoholic chronic pancreatitis: summary of an international workshop on chronic pancreatitis. Pancreas 1997, 14:215-221

13. Kloppel G, Detlefsen S, Feyerabend B: Fibrosis of the pancreas: the initial tissue damage and the resulting pattern. Virchows Arch 2004, $445: 1-8$

14. Freedman SD: New concepts in understanding the pathophysiology of chronic pancreatitis. Int J Pancreatol 1998, 24:1-8

15. Kloppel G, Maillet B: The morphological basis for the evolution of acute pancreatitis into chronic pancreatitis. Virchows Arch A Pathol Anat Histopathol 1992, 420:1-4

16. Haber PS, Keogh GW, Apte MV, Moran CS, Stewart NL, Crawford DH, Pirola RC, McCaughan GW, Ramm GA, Wilson JS: Activation of pancreatic stellate cells in human and experimental pancreatic fibrosis. Am J Pathol 1999, 155:1087-1095

17. Apte MV, Wilson JS: Stellate cell activation in alcoholic pancreatitis. Pancreas 2003, 27:316-320

18. Bachem MG, Zhou Z, Zhou S, Siech M: Role of stellate cells in pancreatic fibrogenesis associated with acute and chronic pancreatitis. J Gastroenterol Hepatol 2006, 21(Suppl 3):S92-S96

19. Apte MV, Wilson JS: Mechanisms of pancreatic fibrosis. Dig Dis 2004, 22:273-279

20. Bachem MG, Schneider E, Gross H, Weidenbach H, Schmid RM, Menke A, Siech M, Beger H, Grunert A, Adler G: Identification, culture, and characterization of pancreatic stellate cells in rats and humans. Gastroenterology 1998, 115:421-432

21. Shek FW, Benyon RC, Walker FM, McCrudden PR, Pender SL, Williams EJ, Johnson PA, Johnson CD, Bateman AC, Fine DR, Iredale JP: Expression of transforming growth factor-beta 1 by pancreatic stellate cells and its implications for matrix secretion and turnover in chronic pancreatitis. Am J Pathol 2002, 160: $1787-1798$

22. Yasuda $\mathrm{M}$, Ito $\mathrm{T}$, Oono $\mathrm{T}$, Kawabe $\mathrm{K}$, Kaku $\mathrm{T}$, Igarashi $\mathrm{H}$, Nakamura T, Takayanagi R: Fractalkine and TGF-beta1 levels reflect the severity of chronic pancreatitis in humans. World J Gastroenterol 2008, 14:6488-6495

23. Adrych K, Smoczynski M, Stojek M, Sledzinski T, Korczynska J, Goyke E, Swierczynski J: Coordinated increase in serum plateletderived growth factor-BB and transforming growth factor-beta1 in patients with chronic pancreatitis. Pancreatology 2011, 11: 434-440

24. Friess $\mathrm{H}, \mathrm{Lu} \mathrm{Z}$, Riesle E, Uhl W, Brundler AM, Horvath L, Gold LI, Korc M, Buchler MW: Enhanced expression of TGF-betas and their receptors in human acute pancreatitis. Ann Surg 1998, 227:95-104

25. Yoo BM, Yeo M, Oh TY, Choi JH, Kim WW, Kim JH, Cho SW, Kim SJ, Hahm KB: Amelioration of pancreatic fibrosis in mice with defective TGF-beta signaling. Pancreas 2005, 30:e71-e79

26. He J, Sun X, Qian KQ, Liu X, Wang Z, Chen Y: Protection of cerulein-induced pancreatic fibrosis by pancreas-specific expression of Smad7. Biochim Biophys Acta 2009, 1792:56-60

27. van Laethem JL, Deviere J, Resibois A, Rickaert F, Vertongen P, Ohtani H, Cremer M, Miyazono K, Robberecht P: Localization of transforming growth factor beta 1 and its latent binding protein in human chronic pancreatitis. Gastroenterology 1995, 108:1873-1881

28. Lieber CS, DeCarli LM: The feeding of ethanol in liquid diets. Alcohol Clin Exp Res 1986, 10:550-553

29. Ceyhan GO, Bergmann F, Kadihasanoglu M, Erkan M, Park W, Hinz U, Giese T, Muller MW, Buchler MW, Giese NA, Friess H: The neurotrophic factor artemin influences the extent of neural damage and growth in chronic pancreatitis. Gut 2007, 56:534-544

30. Fortunato F, Berger I, Gross ML, Rieger P, Buechler MW, Werner J: Immune-compromised state in the rat pancreas after chronic alcohol exposure: the role of peroxisome proliferator-activated receptor gamma. J Pathol 2007, 213:441-452

31. Leask A, Abraham DJ: TGF-beta signaling and the fibrotic response. FASEB J 2004, 18:816-827 
32. Gao HK, Zhou ZG, Li Y, Chen YQ: Toll-like receptor 4 Asp299Gly polymorphism is associated with an increased risk of pancreatic necrotic infection in acute pancreatitis: a study in the Chinese population. Pancreas 2007, 34:295-298

33. Li HG, Zhou ZG, Li Y, Zheng XL, Lei S, Zhu L, Wang Y: Alterations of Toll-like receptor 4 expression on peripheral blood monocytes during the early stage of human acute pancreatitis. Dig Dis Sci 2007, 52:1973-1978

34. Guenther A, Aghdassi A, Muddana V, Rau B, Schulz HU, Mayerle J, Kraft M, Whitcomb DC, Lerch MM, Weiss FU: Toll-like receptor 4 polymorphisms in German and US patients are not associated with occurrence or severity of acute pancreatitis. Gut 2010, 59:1154-1155

35. Miyamoto T, Nakamura $H$, Nagashio $Y$, Asaumi H, Harada M, Otsuki M: Overexpression of Smad6 exacerbates pancreatic fibrosis in murine caerulein-induced chronic pancreatic injuries. Pancreas 2010, 39:385-391

36. Vonlaufen A, Phillips PA, Xu Z, Zhang X, Yang L, Pirola RC, Wilson JS, Apte MV: Withdrawal of alcohol promotes regression while continued alcohol intake promotes persistence of LPS-induced pancreatic injury in alcohol-fed rats. Gut 2011, 60:238-246 